\title{
BEAM-BASED MAGNETIC ALIGNMENT OF THE FINAL FOCUS TEST BEAM*
}

\author{
P. Tenenbaum, D. Burke, R. Helm, J. Irwin, P. Raimondi \\ Stanford Linear Accelerator Center, Stanford University, Stanford, CA 94309 USA \\ K. Oide \\ National Laboratory for High Energy Physics, KEK, Tsukuba, Japan \\ K. Flötrmann \\ Deutsches Electronen-Synchrotron, Hamburg, Germany
}

In order to optimize tunability and backgrounds in linear collider final focus systems, it is necessary to align strong quadrupole and sextupole magnets with beam-based measurements. Algorithms for aligament bave heen uses successfully on the Final Focus Test Beam (FFTB) beamline at SLAC. Quadrupole magnets were aligned using a shunt tecthique, with resolutirns from 50 microds down to 700 nanometers. Sextupole magnets v/ere aligned by boving the magnets transverse to the beam and observing the kick on downstream beam position monitors. This procedure resulced in sexwpple misalignment resolutions of 5 to 20 mictops. All magpets were then moved ioto aligned positions via remotecontrolled stages capable of sub-micron resolution. Letails of the fitling algoritbms, results of the measurement, and potential improvements in the system are discussed.

\section{INTRODUCTION}

Linear colliders operating in the JeV CM exergy range are expected to bave extremely tight a priori aligument tolerances on teir quadrupole and sextupole elements. Misaligned quadrupoles generate dispersion, wbich can dilute the nanometer-sized focused spor; they can also cause the two beams to he steesed out of collision, to sucb an extent that $r$ steering with correctors inuoduces unacceptable disnersion. Misaligned sextupoles can generate normal and skew quadrupole effects, resulting in waist shifts, dispersion, and coupling ( $x$ ' $y$ ) at the $\mathbb{P}$. Fually, any significantly misaligned magoet can create detector backgrounds through aperture imiting in the element itself, or downstrean via steeting.

The Final Focus Test Beam (FFTB) is a prototype linear collider fina: focus designed to focus the $46.6 \mathrm{GeV}$ SLAC beam to a vertical size of 60 nanometers. Tuning studies [1] bave indicaled that the spot size goal can be achieved if the RMS misalignments for quadrupoles and sextupoles do not excoed 100 microns in the horizontal and 30 microns in the vertical. In order to achieve these tolerances, we have developed a beam-based algorithm for measuring the miselignments of all strong quadrupole and sextupole magnets upstream of the Focal Point (FP). The magnets are then moved into aligned positions by remote-controlled stages. This eliminates the noed to shut of the beam and enter the tunnel to correct alignment. reducing the coneornitant risks from changes in the tunnel environment during positioning.

\section{THE FINAL FOCUS TEST BEAM}

\footnotetext{
"Work supported by the Depanment of Energy contract DE-ACO3-76SFO0S IS
}

The optics of the FFTB bave been discussed elsewberein 2]. There are 30 strong quadrupoles upstream of the FP which are subject to beam-based aligument, and 4 sextupoles arranged in 2 families. The beamline contains 40 beam position monitors (BPMs) of a stripliee desigo which are used in the alignment procedure[3]. Eacb quadrupole and sextupole magoet subject to besm-bared alignment is mounled on a remote-controlled stage capabie of independent $x$ and $y$ motion, with positioning nccuracy of under 1 micron[4].

\section{iII. PREPARATION FOR ALIGNMENT}

Prior to the beginning of beam-based alignmedt, the strong sextupole magnets are reduced to a nominal zero value. The eatire line is then standardized. The magnets from the first bend magnet to the end of the line are set to their design values for small-spot operation. The matching guadrupoles upstream of the first bend are set to produce a low-divergence beam at the FP. This setting reduces the beam size througbout mosl of the FFTB, which eases constraints on the shunt range of the quadrupoles, and minimizes beam jitter and BPM background considerations. Tue FFTB enclosure is locked. and allowed to warn up to thennal equilibrium for several days.

\section{QUADRUPOLE ALIGNMENT}

The quadrupole aligameat procedure uses a sbunt technique to measure the offset of the beam ceniroid from the magnetic center of each quad. Each quadrupole upstream of the FP is powered by a separate power supply, eliminating the zeed for shunt or boost supplies to change the quadrupole streugth. The strength range of each quedrupole bas been determined for the low-divergence optics by a series of simulation studies whicb optimize resolution and downstream speriure clearances at eacb serting of every quadrupole.

\section{A. Data Acquisition}

The beamline is aligned in segmęts, each of which contains between 3 and 6 consecutive magnets. For each segwent, the alignment acquisition procedure is as follows:

1. Read in all FFTB-region BPM5 for 100 ortits, average the positions at each BPM over the 100 orbits. This average constitutes the "reference orbit," which is subcracted from all subsequent BPM data at the begirining of the InL This reduces the data used in fitting to differences from the reference orbic which are correlated to quadrupole sbuot

Presented at the 16th IEEE Particle Accelerator Conference (PAC 95) and International Conference on High Energy' Accelerators, Dallas, Texas, May 1.5, 1995 
values to extract the misaligaments. Despite extensive averaging. the refereace orbit may still differ systemalically from the nominal trajectory due to injection or energy offsets. The reference orbit is compared to several subsequent orbits to ensure its conformity before continuing acquisition.

2 Scan each quadrupole in the segment sequentially through its range of strengths. Three setings are used for each magnet, typically the nominal surength and (nominal \pm offset). At each serting, acquire readin; ; from all FFTB BPMs for B-10 pulses (not averaged). During this time, operators watch loss monitors, energy feedback signals, etc., to easure that no errors occur which may contaminate the data. If so, the data for a quadrupole may be re-taken.

3. Once all data for a segment has been acquired successfully, the BPM and magnet strength data are submitted to a fiting rousine, OPTFTT.

\section{B. Fining Algorithm -- OPTFIT}

OPTFT is an online program wbich combines first-order matrix formalisw for ceptroid andor beam matrix transpor with MINUIT function minimization. It uses beamline data (magnet strengtbs, BPM readings, wire scanner measurements) to fit selected parameters of the line (maguet misalignments, strengths, incoming beam matrix).

The program takes as input the data acquired via the SLC dars acquisition system; files describing the beamline devices and the transfer matrices belween them; and a sel of flags which indicate the parameters to be fit. Once this data has been passed to OPTFT, the following steps are followed:

1. The refereace orbit is subtractod from all otber BPM data.

2 For each pulse, the energy variation from the reference orbi: is determined. The FFTB extraction line contains BPMs on either side of a verical permanent bead magnet, and these are used for this computation. The energy and exergy uncerainty of each pulse are stored, and the BPM data used for this step are not used in the main 6 .

3. For eacb pulse, the iscoming jitter $\left(x, x^{\prime}, y^{\prime}, y^{\prime}\right)$ relative to the reference orbit is determined. This is done using BPMs upstream of the first magnet whose aligament is to be fitued, i.c., in a region of nou-varying transfer maerices. The fitted incoming coordinates and their error matrix are stored for each pulse, and the dati used in this step are climinated from the main fil. Because steps 2 and 3 are simple linear fits, matrix inversion (not MINUTT) is used.

4. The dati between the last magnet to be fitted and the energy BPMs are subjected to quality tests. Becouse these BPMs are also in a regiod of invariant transport, the BPM readings can be fitted, pulse by pulse, to a "rack" emanating from the dowastream face of the last fitted magnet. The quality of fit to the "tracks" can indicate BPMs with excessive noise, individual bad teadiogs, etc. These are eliminated from the fit.

5. The errors from 6ring the incoming beam are propagated to each BPM used in the fit, and added in quadrature to the inurinsic 3PM resolution.
6. MINUIT is called. Tbe fit algotithm wul tben minimize $\chi^{2}$ by changing the maget positions and ye-transporting each pulse (using the intial coordjpates determinod atove), then comparing the sesults to the BPM data. The fitted misaligumeols are then retumed, alogg with ugcertainties, the normalized $\chi^{2}$, and the contribution to $\chi^{2}$ from each BPM.

\section{Refinements to the Procedure}

Early experiments indicated that naively implementing the corrections recommended by OPTFTT was not satisfactory in all cases: frequently the misaligament of an upsueam quadrupole served to kick the beam onto the line of the remaining magnets. In this case, simply moving the magnets would have forced us to move the entire FFTB line onto the arbitrary line of the incoming beam. A fit option was added in which the last quadrupole of a segmedt is defined to be "aligned," and a kick angle is fitred at the upstream end of the segment. This dramatically improved our ability to converge, especially in the beam matching region upstream of the first bend.

Other experinents showed that the guality of the fit was deteriorating as the area of interest moved dowastream, as indicated by monotonically increasiog normalized $\chi^{2}$ values. This was traced to upstream magnets losing bysteresis, usually at the end of a scan (whed set back to their original values). The magnets were then required to "mini-standardize," i.e., when the magnels are cbanged in a direction opposite to their bysteresis curves, the power supplies automatically overshoot the new set point by $5 \%$, so the set point is approached from the correct direction. The dilution of fit quality was nearly eliminated by this refioement.

\section{Results}

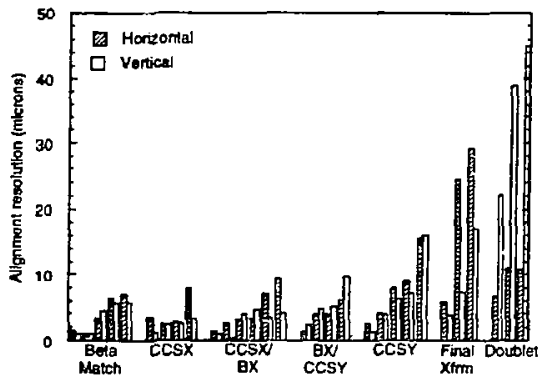

Figure 1. Resolution of quadrupole alignmeal technique for all FFTB magnets upstream of FP.

Figure 1 shows the achieved resolutions of the fining procedure. These represeat the resolution of the distance trom the quadrupole center to the athitrary line of the incoming beam. Each quadnupole in the segment introduces a kick to 
the nominal trajectory, whose magnitude is uncertain due to the uncertainty in the quadrupole's alignment. The propagation of this kick is included in the fit of the downstream quadrupoles. Consequenty, the resolution of the method degrades from upstream quadrupoles to downstream within a given segment. The monotonic loss of resolution from upstrean segments to dowastream segments is due w the decressing number of BPMs downstream of the fitted magnets.

\section{SEXTUPOLE ALICJNMENT}

The SLC Final Focus performs CCS sextupole alignment by varying their sextupole families in strength and observing changes in waist, dispersion and coupling at the IP[S]. This technique relies on IP single-beam size monitors, which can be difficulı to use in a linear collider final focus. The FFTB aligament rechnique, by contrast, relies only on BPMs and magnet movers, and can be completed before small-spot tuni begins.

Once all the quadrupoles bave beed aligoed, the CCS sextupoles are turned on to a strong value (integrated second derivative $=33.000 \mathrm{kG} / \mathrm{m}$ ). The sextupole is thell seanned in position, via its mover, over the full range of the mover in either $x$ or $y$. The thin lens kick of a sextupole magnet is of the form:

$$
B_{y}=K_{5}\left(x^{2}-y^{2}\right) \text {. }
$$

Consequently, the position on $\varepsilon$ downstream BPM will vary quadratically in $x$ as a function of the mover ( $x$ or $y$ ) position. The downstream BPM values can then be fit to a parabola:

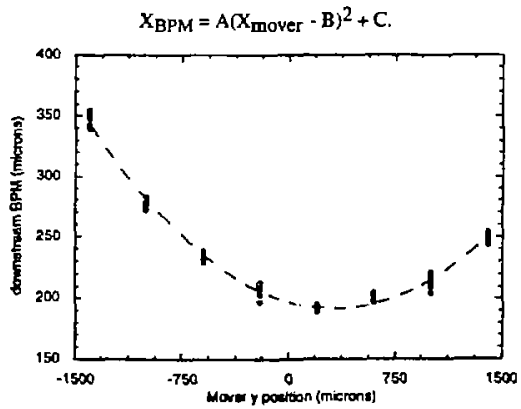

Figure 2. Reading of a downstream BPM vs sextupole mover position. Note that both $x$ and $y$ mover scans produce a quadratic borizontal kick al the downsteam BPM.

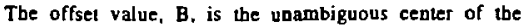
sextupole magne!, i.e., the point at which the magnetic gradient vanishes. The sextupole may then simply be set to this position. Figure 2 shows sucb a quadratic form for a BPM vs. sextupole mover sean, and Figure 3 shows the achieved resolution of this procedure for all 4 FFTB CCS sextupoles.

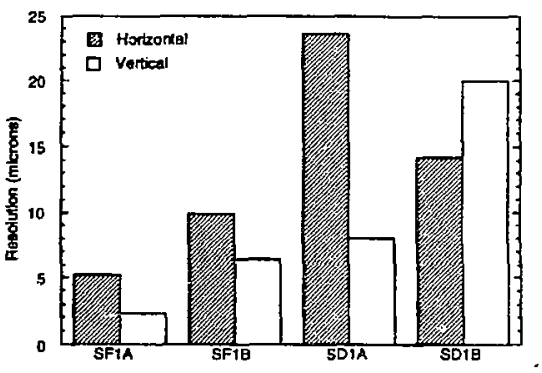

Figure 3. Resolution of sextupole aligument wechnique for all four CCS sexupoles.

\section{POSSIBLE MMPROVEMENTS}

The incoming trajectories for the aligoment of the upstream quadrupoles in the FFTB is determined by a pair of BPMs, separated by 85 meters. The first of these is a lowresolution device, rather than a high-resolution FFTB BPM. Replacing this BPM would improve convergence of these magnets. Since the upstream quadrupoles are used for beam matching and changing the IP divergence, this would be a significant improvement.

\section{ACKNOWLEDGMENTS}

The authors would like to thank Lee And Yasukawa for writing the Quad Alignment incerface sofiware in the SLAC contol system.

\section{REFERENCES}

[1] K. Oide. "Design of Optics for the Final Focus Test Beam at SLAC." Proc. of the IEEEPart Acc. Couf. 1989.

[2] G. Roy, "Analysis of the Optics of the Final Focus Test Beam Using Lie Algebra Based Techniques." SLAC. Repon-397 (1992).

[3] H. Hayano at al, "Higb Resolution BPM for FFTB." Nucl. Inst. Methods A320:47-52 (1992).

[4] G. Bowden et al, "Precision Magnet Movers for the Final Focus Test Beam." SLAC-PUB-6132 (1994).

[5] P. Emma et al, "Beam-Based Alignment of the SLC Final Focus Sextupoles," Proc. of the IEEE Part Acc. Conf., 1993. 